\title{
Medial Temporal Lobe Amnesia: Gradual Acquisition of Factual Information by Nondeclarative Memory
}

\author{
Peter J. Bayley² and Larry R. Squire ${ }^{1,2,3,4}$ \\ ${ }^{1}$ Veterans Affairs Medical Center, San Diego, California 92161, Departments of ${ }^{2}$ Psychiatry, ${ }^{3}$ Neurosciences, and \\ 4Psychology, University of California, San Diego, La Jolla, California 92093
}

\begin{abstract}
Most amnesic patients with damage to the medial temporal lobe retain some capacity to learn new information about facts and events. In many cases, the learning appears to depend on a residual ability to acquire conscious (declarative) knowledge. We have studied the capacity for semantic (fact) learning in the profoundly amnesic patient E.P., who has extensive damage limited primarily to the medial temporal lobe. E.P. was presented with factual information (novel three-word sentences) during 24 study sessions across 12 weeks. E.P. performed much more poorly than controls but demonstrated unmistakable improvement across the sessions, achieving after 12 weeks a score of $18.8 \%$ correct on a cued-recall test and $64.6 \%$ correct on a two-alternative, forced-choice test. Unlike controls, E.P.'s learning was not accompanied by conscious knowledge about which answers were correct. He assigned the same confidence ratings to his correct answers as his incorrect answers. Moreover, on the forced-choice test his response
\end{abstract}

Declarative memory affords the capacity for conscious recollections about facts and events and depends on the integrity of the medial temporal lobe memory system (Squire, 1992; Milner et al., 1998; Eichenbaum and Cohen, 2001). Damage to this system impairs both fact and event (semantic and episodic) learning. Nevertheless, memory impairment is rarely absolute, and some residual learning capacity typically remains. For example, it has often been observed that amnesic patients can acquire some new factual information (termed semantic knowledge) (Glisky et al., 1986a,b; Tulving et al., 1991; Hayman et al., 1993; Hamann and Squire, 1995; Kitchener et al., 1998; Verfaellie et al., 2000; Van der Linden et al., 2001; Westmacott and Moscovitch, 2001).

In patients with large medial temporal lobe lesions and severe amnesia, the capacity for new semantic learning seems quite limited (patient H.M.: Marslen-Wilson and Teuber, 1975; Gabrieli et al., 1988; Hood et al., 1999; patient S.S.: Verfaellie et al., 1995; patient E.P.: Reed and Squire, 1998). When the damage is more restricted and the amnesia correspondingly less severe, considerable semantic learning is possible (Hamann and Squire, 1995; Kitchener et al., 1998; Reed and Squire, 1998; Verfaellie et al., 2000; Van der Linden et al., 2001), although the degree of

\footnotetext{
Received Feb. 1, 2002; revised April 15, 2002; accepted April 15, 2002.

This work was supported by the Medical Research of the Department of Veterans Affairs, National Institute of Mental Health Grant MH24600, and the Metropolitan Life Foundation. We thank Dr. Craig Stark and Joe Manns for their helpful comments and Shauna Stark and Joyce Zouzounis for assistance.

Correspondence should be addressed to Dr. Larry Squire, Veterans Affairs Medical Center (116A), 3350 La Jolla Village Drive, San Diego, CA 92161. E-mail: Lsquire@ucsd.edu.

Copyright (C) 2002 Society for Neuroscience $\quad 0270-6474 / 02 / 225741-08 \$ 15.00 / 0$
}

times were identical for correct and incorrect responses. Furthermore, unlike controls, he could not respond correctly when the second word in each sentence was replaced by a synonym. Thus, what E.P. learned was rigidly organized, unavailable as conscious knowledge, and in all respects exhibited the characteristics of nondeclarative memory. Thus, factual information, which is ordinarily learned as declarative (conscious) knowledge and with the participation of the medial temporal lobe, can be acquired as nondeclarative memory, albeit very gradually and in a form that is outside of awareness and that is not represented as factual knowledge. We suggest that E.P.'s learning depended on a process akin to perceptual learning and occurred directly within neocortex.

Key words: memory; hippocampus; medial temporal lobe; declarative memory; nondeclarative memory; semantic learning; amnesia; patient E.P.

learning seldom approaches what can be achieved by normal individuals (for a single case that performed well, albeit still poorer than controls, see Van der Linden et al., 2001; for the possibly special case of developmental amnesia, see VarghaKhadem et al., 1997; Baddeley et al., 2001).

The question arises: when memory-impaired patients do acquire factual knowledge, what kind of learning occurs? Is learning supported by a residual capacity for declarative memory, or is some other (nondeclarative) memory system able to support performance?

In many cases when fact learning has been studied, patients appear to acquire declarative knowledge. For example, although learning is impaired, the knowledge that is expressed is consciously accessible (Reed et al., 1997; Westmacott and Moscovitch, 2001), flexible, and accessible to indirect cues, like the knowledge acquired by normal individuals (Shimamura and Squire, 1988; Hamann and Squire, 1995). In these cases, the residual ability of amnesic patients to acquire declarative memory may be supported by structures within the medial temporal lobe that remain intact. [It has also been suggested that declarative knowledge might be acquired gradually by the neocortex (Tulving, 1991)].

The question remains whether the acquisition of factual knowledge can ever be supported by nondeclarative memory. Declarative and nondeclarative knowledge have different characteristics (Cohen, 1984; Squire, 1992; Eichenbaum and Cohen, 2001). Accordingly, if nondeclarative learning of factual information is possible, the acquired knowledge should be qualitatively different from the knowledge acquired by normal individuals. Alterna- 
Table 1. Memory performance of patient E.P.

\begin{tabular}{|c|c|c|c|c|c|c|c|c|}
\hline \multirow[b]{2}{*}{ E.P. } & \multirow{2}{*}{$\begin{array}{l}\begin{array}{l}\text { Diagram } \\
\text { recall }\end{array} \\
0\end{array}$} & \multicolumn{3}{|c|}{ Paired associates } & \multirow{2}{*}{$\begin{array}{l}\text { Word } \\
\text { recall } \\
(\%)\end{array}$} & \multirow{2}{*}{$\begin{array}{l}\text { Word } \\
\text { recognition } \\
(\%)\end{array}$} & \multirow{2}{*}{$\begin{array}{l}\begin{array}{l}\text { Words } \\
(50)\end{array} \\
24\end{array}$} & \multirow{2}{*}{$\begin{array}{l}\begin{array}{l}\text { Faces } \\
(50)\end{array} \\
28\end{array}$} \\
\hline & & 0 & 0 & 0 & & & & \\
\hline Controls & 20.6 & 6.0 & 7.6 & 8.9 & 71.0 & 97.4 & 48.4 & 41.9 \\
\hline
\end{tabular}

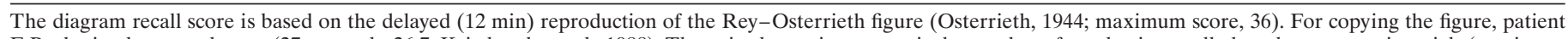

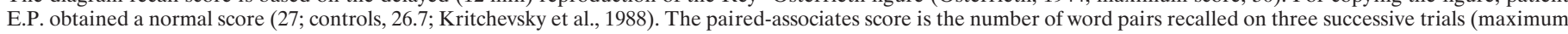

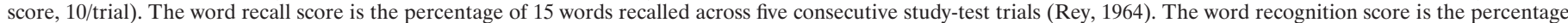

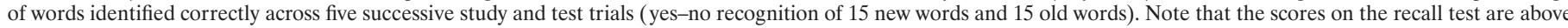

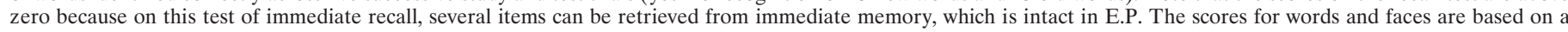

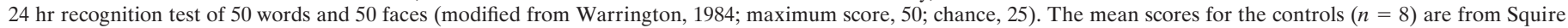
and Shimamura (1986).

tively, humans may be so dominated by specific learning strategies that memory systems cannot readily substitute for one another. Studies in rats and monkeys suggest that after lesions to the hippocampus or related structures, learning of hippocampusdependent tasks can still succeed, albeit slowly (Eichenbaum et al., 1989, 1990; Saunders and Weiskrantz, 1989) and that what is learned is less flexible and less accessible in novel situations than is normal memory. Similarly, it has been reported that information acquired gradually by amnesic patients after extended training can be somewhat inflexible and hyperspecific (Milner et al., 1968; Glisky et al., 1986a,b). These findings might reflect the operation of nondeclarative (skill-like) memory, residual declarative (fact-like) memory, or some combination.

We have studied the capacity for semantic (fact) learning in the profoundly amnesic patient E.P. (Stefanacci et al., 2000). After patient H.M. (Scoville and Milner, 1957), E.P. is the only severely impaired amnesic patient known to us for whom detailed neuroanatomical and neuropsychological data are available and whose damage is primarily limited to the medial temporal lobe. The results indicate that factual information, which ordinarily would be acquired as declarative knowledge, can also be acquired as nondeclarative memory, albeit very gradually. Importantly, as nondeclarative memory the information that was acquired was rigidly organized and unavailable as conscious knowledge.

\section{MATERIALS AND METHODS}

\section{Participants}

Amnesic Patient E.P. E.P. is a 79-year-old retired laboratory technician with 12 years of education who, in 1992, developed profound anterograde and retrograde amnesia after contracting viral encephalitis (see Table 1 for neuropsychological data). His IQ is 98 [Wechsler Adult Intelligence Scale-III (WAIS-III) (Wechsler, 1997)]. On the Wechsler Memory ScaleRevised (WMS-R) (Wechsler, 1987), he scored 94, 57, 82, 61, and 56 on the attention-concentration, verbal memory, visual memory, general memory, and delayed memory subtests, respectively. Each of these subtests yields mean scores of 100 in the normal population with an SD of 15 .

Detailed magnetic resonance imaging revealed extensive damage to the medial temporal lobe bilaterally (Stefanacci et al., 2000). The damage extends caudally from the temporal pole to involve bilaterally all of the amygdaloid complex, all of the entorhinal and perirhinal cortices, and much of the parahippocampal cortex (20\% on the left and $60 \%$ on the right). In addition, nothing remains of the hippocampus except a small tag of abnormally appearing vestigial tissue on each side that amounts to $\sim 10 \%$ of the hippocampal volume. The abnormal appearance of this tissue and the absence of entorhinal cortex (which originates the major cortical afferents to the hippocampus) make it quite unlikely that the remnant tissue is functional. At the level of the amygdala, the damage extends lateral to the parahippocampal gyrus to include the anterior fusiform gyrus ( $40 \%$ on the left, $53 \%$ on the right). Finally, the lateral temporal cortex and the insula are somewhat reduced in volume bilaterally (19 and $13 \%$ reductions, respectively).
Controls. Controls $(n=4)$ were employees or volunteers at the San Diego Veterans Affairs Healthcare System and matched patient E.P. with respect to gender, age (mean, 75.5 years; range, 72-80), and education (mean, 12.3 years).

\section{Materials}

Test of semantic learning. Sixty three-word sentences were constructed (subject-verb-object, e.g., shark killed octopus) based on sentences used previously (Tulving et al., 1991; Hamann and Squire, 1995). Of these, 35 were identical to sentences from our earlier study (Hamann and Squire, 1995). Forty-eight sentences were used for training, four additional sentences were used as practice items at the beginning of each session, and the remainder were used to replace sentences that were completed correctly at pretest (see below).

The object of each sentence (the target word) was always a 5-8 letter noun (frequency, 1-99 per million; Thorndike and Lorge, 1944), and was difficult to guess given the subject and verb. A foil word was also generated for each of the 60 target words and was used in a forced-choice recognition memory test. Foil words were intended to provide an equally plausible alternative to the target word. They were chosen using the same selection criteria as target words, with the additional constraint that the foil word and its corresponding target word began with different letters.

The task was presented on a computer. For study sessions, each three-word sentence was presented with the first and last words capitalized and the second word in lowercase (e.g., "SPEECH caused LAUGHTER"). For the pretest and for the cued-recall test, the sentence frame was presented alone, and the third word of the sentence was replaced by question marks (e.g., "SPEECH caused ???").

\section{Procedure}

Overview. Patient E.P. and controls followed a "study only" or "errorless learning" procedure (Tulving et al., 1991; Wilson, 1992; Hamann and Squire, 1995). After a pretest to eliminate the few sentences that were completed correctly, study sessions were given in which participants saw each of the 48 training sentences twice. Controls were given a total of two study sessions (one session per week for 2 weeks for a total of four training trials). E.P. was given a total of 24 study sessions on 24 different days (two sessions per week for 12 weeks for a total of 48 training trials). Retention in the control group was tested twice, $7 \mathrm{~d}$ after the second of the two study sessions and again after 5-6 months (mean, 169 d). E.P.'s retention was tested 3 and $4 \mathrm{~d}$ after the eighth study session (T1, T2), 4 and $5 \mathrm{~d}$ after the $16^{\text {th }}$ study session (T3, T4), and 3 and $4 \mathrm{~d}$ after the $24^{\text {th }}$ study session (T5, T6). Finally, E.P. was tested on 2 consecutive days $\sim 3$ months after T6 $(92 \mathrm{~d})$.

The retention tests assessed the ability to remember the target word when the sentence frame was given as a cue. On each day of retention testing, a cued-recall test was presented first. Specifically, each sentence frame was presented, and participants tried to recall the target word. The cued-recall test was followed by a two-alternative forced-choice test in which each sentence frame was presented together with two possible completions, and participants were asked to select the target word. E.P. (but not the controls) was also given two additional tests at the conclusion of the final visit after 12 weeks of study. The first test was identical to the cued-recall test, except that the sentence frames were spoken by the examiner instead of presented on a computer. In addition, E.P. was asked to indicate how confident he was (on a 1-5 scale) that his answer was correct. The second test was also identical to the cued-recall test, except 
that the second word of the sentence frame was replaced by a synonym (control data were taken from Hamann and Squire, 1995).

Pretest. The purpose of the pretest was to identify sentence frames that could be completed with the correct target word in the absence of study. Participants were instructed as follows: "I'm going to show you words on the screen that could be the first two words of a sentence. For each of the two words, I'd like you to think of a third word to complete the sentence in a sensible way."

Sixty sentence frames were presented one at a time in the same order to all participants. The first four sentence frames were practice items. Participants were given $15 \mathrm{sec}$ to suggest a word to complete each sentence. After elimination of sentences that were completed correctly, 48 training sentences (plus the four practice sentences) were assembled for each participant.

Study. Participants were instructed as follows: "You will see some sentences that I want you to try to remember. Each sentence has three words in it. For example, you might see a sentence like this: 'MUSICIANS formed QUARTET.' When a sentence appears on the screen, read the sentence aloud and think about what it means. Some time later, I will show you the words 'MUSICIANS formed', and I will ask you to remember the word that went with it, in this case, 'QUARTET'".

All participants received nearly the same 48 sentences for study, plus the four practice sentences. (E.P. received on average 46.8 of the same sentences that the controls received). The four practice sentences and 48 study sentences were presented twice per study session, always in the same order. Sentences were presented one at a time, and the participant was asked first to read the sentence aloud and then to rate it for meaningfulness on a 1-5 scale. A pause of 5-10 min intervened before the sentences were presented a second time.

After participants read the sentence, it remained on the screen, and the rating question appeared beneath it: "How much sense does this sentence make?" Participants rated the meaningfulness of the sentence by pressing one of five keys on the keyboard. The leftmost key was labeled "very little" and the rightmost key was labeled "a lot." Three seconds after a key press, the screen went blank, and the next sentence appeared when the experimenter judged the participant to be ready.

Cued recall test. Sentence frames were presented on the computer screen in the same order as in the study sessions. Participants were instructed as follows: "I'm going to show you two words on a screen which are the first two words of a sentence that I have shown you before. For each of the two words, I would like you to try and remember the last word of the sentence." Guessing was encouraged, and participants were given up to $15 \mathrm{sec}$ to generate a response. No feedback was given, and the next item was presented when the participant was ready.

Forced-choice recognition test. Sentence frames were presented on the computer screen in the same order as in the study sessions, together with the target word and the foil word. Participants were instructed as follows: "I'm going to show you words on the screen that are the first two words of a sentence that I have shown you before. For each of the two words, try and remember the word that completed the sentence. To make it easier for you, I will give you a choice of two words and I want you to choose the word that you remember completed the sentence." Participants were given up to $15 \mathrm{sec}$ to press a key indicating their choice, and response times were recorded. No feedback was given. After the key press, the screen went blank, and the next sentence frame was presented when the participant was ready.

Obtaining a baseline score for the forced-choice recognition test. To determine how individuals would perform on the forced-choice test in the absence of any training, we presented the same 48 sentences that were given to E.P. to a group of 10 naive controls (mean age, 68.2 years; range, 57-79; mean education, 16.3 years). Participants were asked to choose which word (target or foil) they thought we might have used to complete that sentence in constructing a study of memory: "Please guess which word we used to complete each sentence in our other study. You should not necessarily choose the most sensible word, but simply guess which one of the words you think we used in our memory study."

Spoken cued-recall transfer test. Sentence frames were read aloud to E.P. in the same order as in the study sessions, and he was asked to recall the target word. In all other respects, this test was the same as the first cued-recall test (above).

After making his response (E.P. offered an answer for all 48 questions), E.P. was asked to rate his confidence using a five-point scale. One end of the scale was labeled "pure guess," and the other end was labeled "very sure." "I want you to tell me how sure you are that your answer is correct, that the word is the one that I showed you on the computer screen before. If you are very sure that your answer is correct then choose 5. If you don't know the correct answer and are making a pure guess, then choose 1 . The higher the number, the more sure you are. Be sure to use the whole scale from 1 to 5 when making your confidence judgments."

No feedback was given, and the next item was presented when he was ready.

Synonym test. This test was the same as the cued-recall test except that the second word of each sentence frame was replaced by a synonym (e.g., "Venom caused ???" was changed to "Venom induced ???"). E.P. was instructed as follows: "Some of the words that you will see to prompt your memory will be different from the ones you saw before but will be similar in meaning. Try to remember what the word was that you had studied as the last word of the sentence, even if the exact wording of the sentence has been changed."

E.P.'s results were compared with the results from a group in our previous study (Hamann and Squire, 1995; "CON-DELAY2" group, $n=$ 12). This group studied 40 three-word sentences (two "study-only" trials were given), and they were tested with modified sentences 3-5 d later. Twenty-nine of the 40 study sentences were the same as ones given to E.P. For 16 of these sentences, the second word was replaced with the same synonym as in the present study. For the remaining 13 sentences, the second word was replaced with the same synonym as in the present study, and in addition the first word was also replaced with a synonym.

\section{RESULTS}

\section{Study}

The meaningfulness scores generated during the study sessions were similar for E.P. (mean, 3.6) and the controls (range, 3.7-4.2).

\section{Cued-recall pretest}

E.P. generated a word to complete 59 of the sentence frames (controls, 60.0). One of E.P.'s responses was correct (controls: mean, 1.5; range, $0-2$ ).

\section{Cued-recall tests}

After two study sessions, the control group recalled an average of $49.5 \pm 11.9 \%$ of the 48 target words (Fig. $1 A$ ). E.P. was markedly impaired at learning the target words. After 4 weeks of study (eight sessions), he recalled one word on the first retention test (T1) and two words on the second retention test (T2). Thus, across both tests, his recall score after eight study sessions (3.2\%) was far below the $49.5 \%$ score that controls achieved after only two study sessions. Nevertheless, despite his inability to learn scarcely anything during the first 4 weeks of study, E.P. demonstrated unmistakable improvement as the study sessions continued. Thus, he averaged $11.5 \%$ correct on T3 and T4, after 8 weeks of study, and $18.8 \%$ correct (nine words correct/48) on T5 and T6 after a total of 12 weeks of study. The linear trend across the three study-test intervals (T1 and $\mathrm{T} 2, \mathrm{~T} 3$ and $\mathrm{T} 4, \mathrm{~T} 5$ and $\mathrm{T} 6$ ) was significant $\left(F_{(1,5)}=17.2 ; p<0.02\right)$. E.P. tended to be consistent in his answers across the test sessions. That is, on each cued-recall test, he tended to produce the same target words that he had produced on earlier tests, plus additional target words that he had learned since the last test. Across six tests (T1-T6), E.P. recalled 16 of the 48 target words on at least one occasion, and on the sixth test session (T6) he recalled 11 of these 16 target words.

\section{Forced-choice tests}

The 10 controls who took the forced-choice test in the absence of previous study identified $46.9 \pm 2.9 \%$ of the target words. This value was taken as a baseline score with which to compare the performance of E.P. and his controls. Figure $1 B$ shows that controls correctly identified $89.6 \pm 5.6 \%$ of the target words after their two study sessions (single sample $t$ test, $t(3)=16.0 ; p<$ .001). In contrast, E.P. scored 58.3\% correct after eight study sessions (T1 and T2), 64.6\% correct after 16 study sessions (T3 

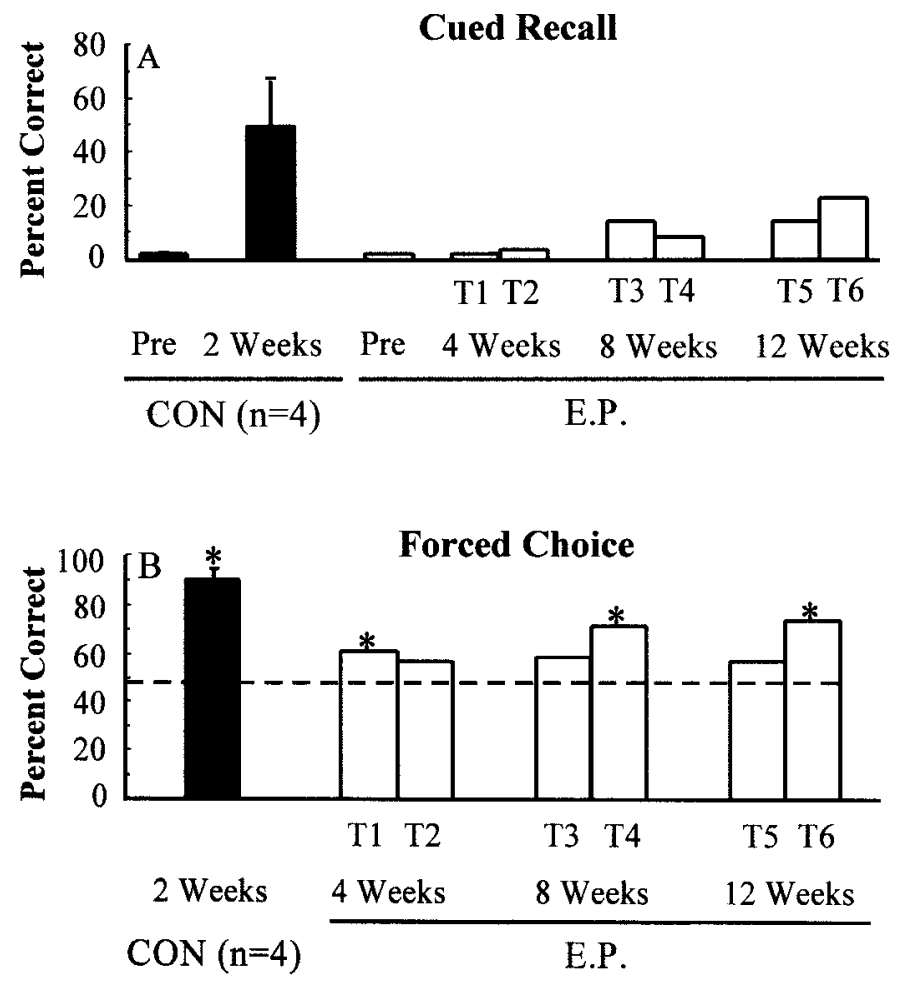

Figure 1. Controls (CON, black bars, $n=4)$ and E.P. (open bars) studied 48 three-word sentences (e.g., "Shark killed octopus"). Retention tests were given to controls after 2 weeks of study ( 1 session per week, 2 training trials per session) and to E.P. on 2 consecutive days (T1-T6) after each 4 week study period ( 2 sessions per week, 2 training trials per session). $A$, Percent correct cued recall of target words in response to the first two words in each sentence. Performance is shown for the pretest (Pre, before study) and after each study period. B, Percent correct forcedchoice recognition when the first two words of each sentence were presented together with two possible target words. Performance is shown after each study period. The dashed line shows the score obtained by a group $(n=10)$ that received no study. Error bars show SEM. Asterisks indicate significant difference versus the no-study group $(p<0.05)$.

and T4), and $64.6 \%$ correct after 24 study sessions (T5 and T6). Although his performance was much poorer than that of the controls, his score was measurably above chance at each study-test interval (binomial test, $p$ values $<0.02$; the results were the same when $50.0 \%$ was taken as chance performance, except that in this case E.P. was not above chance at sessions T1 and T2). In a previous study of recognition memory for repeatedly presented scenes (Reed et al., 1997), E.P. failed to score above chance, but received considerably less repetition of the study material than in the present case.

As in the case of his cued-recall performance, E.P. tended to be consistent in his answers on the forced-choice tests across the sessions. Across six tests (T1-T6), he chose correctly 43 of the 48 target words on at least one occasion. On the sixth test session (T6), he correctly chose 35 of these target words.

The time needed to make each choice was also recorded in each test session (for controls, after 2 weeks of study; for E.P., in sessions T1-T6) (Fig. 2). One control made no errors and could not be included in this analysis. The remaining three controls were faster when they made correct choices (mean, $3.0 \pm 0.5 \mathrm{sec}$ ) than when they made incorrect choices (mean, $5.5 \pm 1.2 \mathrm{sec})(t$ test, $t(2)=3.8, p=0.06)$, consistent with previous findings of faster response times for correct versus incorrect responses in

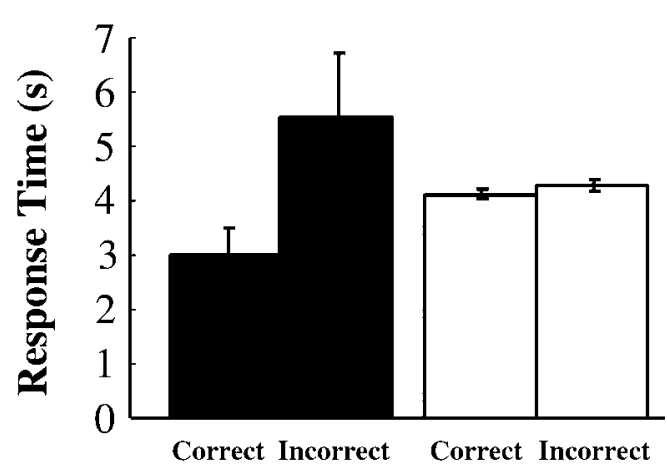

Figure 2. Response latencies for correct and incorrect choices in the forced-choice recognition test. For controls (black bars, $n=4$ ), the score was the mean latency for correct and incorrect responses on the 48-item test. Brackets show the SEM of the four scores. For E.P. (open bars), the score was his mean latency across all six forced-choice tests (T1-T6). Error bars show SEM for all his responses.

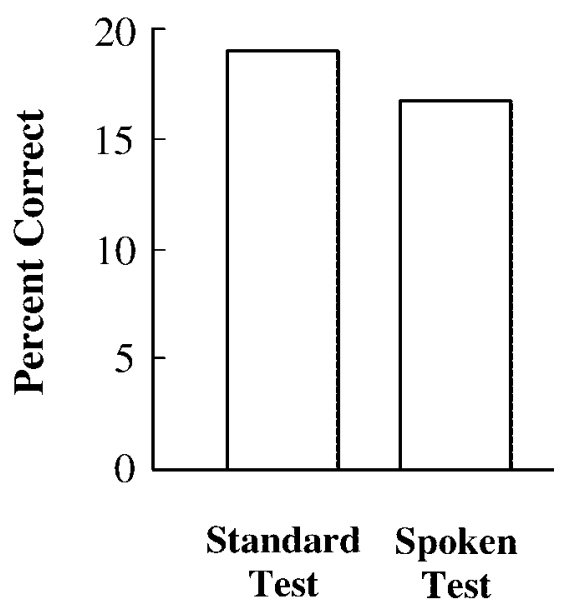

Figure 3. Cued recall performance of E.P. after 12 weeks of study (24 sessions). In the Standard Test, the 48 two-word sentence frames were presented on a computer screen. The score is the average of two tests (T5 and T6 in Fig. 1A). The Spoken Test was given after the Standard Test at T6. In the Spoken Test, the sentence frames were read aloud by the experimenter.

forced-choice recognition memory tasks (Pike et al., 1977). In contrast, E.P.'s response times were nearly identical for correct and incorrect responses (mean, $4.1 \pm 0.1 \mathrm{sec}$ vs mean, $4.3 \pm 0.1$ $\mathrm{sec}$ ). Despite the fact that E.P.'s performance was unequivocally above chance, these data suggest that he was unaware of when he was making correct and incorrect choices.

\section{Spoken cued-recall test}

The ability of E.P. to use his knowledge of the sentences flexibly was assessed on the sixth test day (T6) after all 12 weeks of study. Figure 3 shows that E.P. was able to recall $16.7 \%$ of the target words (eight words) when the sentence frames were read aloud. This score is similar to the $18.8 \%$ average score he obtained on the standard test when the sentence frames were presented on the computer (also see T5 and T6) (Fig. 1 $A$ ).

E.P. rated how confident he was in his answers on the spoken cued-recall test using a five-point scale. His mean confidence rating for correct answers was $3.22 \pm 0.12$ and for incorrect answers was $3.25 \pm 0.31$. Thus, he was no more confident of his correct answers than of his incorrect answers. These results for E.P. contrast with what is typically observed in less severely 


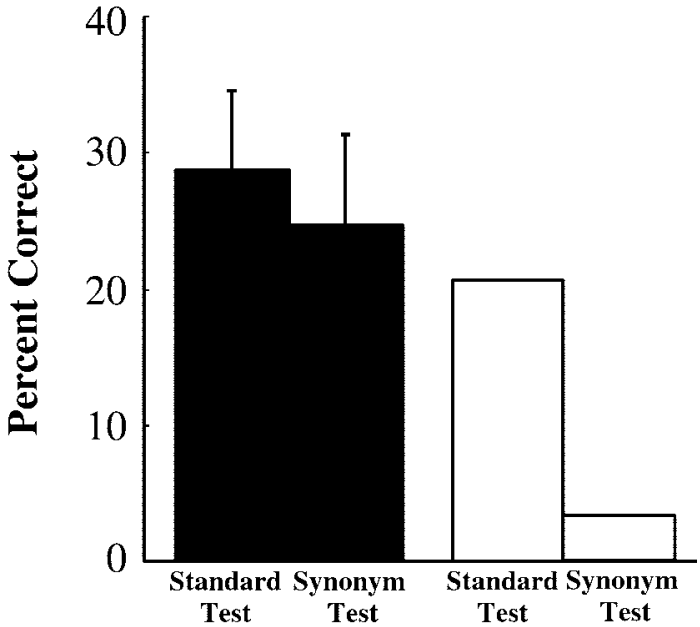

Figure 4. Cued recall performance of target words by controls (black bars, $n=12$ ) and E.P. (open bars) in response to the first two words of each three-word sentence. The score for E.P. is the average of two tests (T5 and T6). Data are for 29 of 48 studied sentences that were given to both E.P. and controls (control data, $n=12$; from Hamann and Squire, 1995). In the Standard Test, given to E.P. after 24 sessions of study and to controls after 1 session of study, two-word sentence frames were presented (e.g., "Venom caused ???"), and subjects responded with the target word (fever). In the Synonym Test for E.P., the second word of each sentence frame was replaced by a synonym (e.g., "Venom induced ???"). For the controls, the same synonyms were used for 16 of the sentence frames. For the remaining 13 sentence frames, both words of the sentence frame were replaced by synonyms (e.g., "Venom caused ???" was replaced by "Poison induced ???"). Error bars show SEM.

impaired amnesic patients and in healthy controls; namely, that confidence ratings are higher for correct answers than incorrect answers (Shimamura and Squire, 1988; Reed et al., 1997). Thus, E.P. did not appear to distinguish between his correct and incorrect answers.

\section{Synonym test}

Figure 4 shows the results when the second word of each sentence frame was replaced by a synonym. Data were taken from 29 of the 48 sentence frames that were given to both E.P. and controls $(n=$ 12; from Hamann and Squire, 1995). Substituting a synonym for the second word in the sentence frame markedly disrupted E.P.'s performance. He scored $20.7 \%$ correct on the standard test when the original sentence frames were used as cues (on T5 and T6), but could recall correctly only one of the 29 target words after the sentences were changed. His performance was identical (one target word recalled) when all 48 sentence frames were scored. In contrast, the control group performed almost as well in the synonym condition ( $24.7 \%$ correct) as in the standard condition (28.7\%), despite the fact that for 16 of the 29 sentences both words of the sentence frame were replaced by synonyms. Thus, the controls were able to use their knowledge flexibly, even when both words of the sentence frame were replaced by synonyms. In contrast, E.P. could sustain his performance only when the words in the sentence frame were exactly the same words that had been studied. When the second word in the sentence frame was replaced by a synonym, his performance (one correct response) was no better than during the pretest (Fig. $1 A$ ) before he had studied any sentences.

\section{Long-term retention}

To determine the stability of what was learned, the ability to remember the target words was also assessed several months after the completion of training. E.P. was tested $\sim 3$ months after the last of his 24 training sessions, and three controls (one was unavailable for testing) were tested 5-6 months after their final study session (range, 160-179 d). On two cued-recall tests on 2 consecutive days, E.P. recalled an average of $10.4 \%$ of the 48 target words (five words correct). The target words that he correctly produced had all been recalled on previous tests. The controls, who had received only two sessions of training 5-6 months earlier, performed similarly to E.P. (3.7 words correct, $7.6 \% \pm 0.7 \%$ correct). The results were quite different on the two forced-choice tests. E.P. correctly chose only $57.3 \%$ of the target words (above chance levels; binomial test, $p<0.03$ ), whereas the controls averaged $81.3 \pm 5.3 \%$ correct $(p<0.01)$. Thus, E.P. and the controls performed similarly on the cued-recall test, but the control group performed much better on the forced-choice test.

It is also notable that E.P. frequently exhibited a type of error on the forced-choice tests that was rare in the control group. On 11 occasions, across all the retention tests, he failed to recognize a word that he had recalled correctly in the immediately preceding cued-recall test. One control made this error on one occasion.

\section{Other observations}

During the study sessions, E.P. was attentive and cooperative and often commented on the study sentences as they were shown to him. Usually, his comments related the study sentence to his remote past, and the same comments tended to be repeated across study sessions. For example, when presented with the sentence "TRAIN frightened KANGAROO," he regularly commented that he had visited Australia during his time in the Merchant Marines and that this kind of incident could indeed occur.

During the test sessions (T1-T6), E.P. never gave any indication that he was familiar with the test materials. Two observations merit comment. First, when he was presented with sentence frames during the cued-recall and forced-choice tests, E.P. never stated that he remembered the target word and would occasionally ask whether he had been shown the sentence before. When he was told that he had in fact seen a sentence before, E.P. consistently expressed surprise and remarked that he must be performing badly on the test. Second, during the cued-recall and forcedchoice tests, E.P.'s behavior did not noticeably differ for correct and incorrect answers. That is, neither his comments, his tone of voice, nor his manner of response suggested that he had any awareness that he sometimes was producing the correct answer.

\section{DISCUSSION}

Patient E.P. has extensive bilateral damage to the medial temporal lobe and has exhibited no capacity for declarative memory on any of the numerous tests of delayed recall and recognition that he has been given during the past several years. For example, in a series of 42 different tests of recognition memory, he averaged $49.3 \%$ correct $($ chance $=50 \%)($ Stefanacci et al., 2000). Nevertheless, in the present study, E.P. demonstrated considerable learning of new information (novel three-word sentences). Although his performance was well below the level achieved by controls, he improved gradually and unmistakably across 12 weeks and 48 training trials, as measured by both cued-recall and forced-choice procedures. Furthermore, some memory of what had been learned persisted for as long as 3 months after the last training trial.

Learning in the present study followed a study-only procedure in which three-word sentences were studied but without an op- 
portunity to make errors during training. This procedure has been found to facilitate learning in memory-impaired patients and to be superior to conventional study-test methods (Tulving et al., 1991; Wilson, 1992; Hayman et al., 1993; Hamann and Squire, 1995). In an earlier study (Hamann and Squire, 1995), E.P. failed to learn under the study-only procedure but was given only eight repetitions of each sentence. The present study shows that as many as 32 repetitions are needed for E.P. to exhibit learning (Fig. 1, T3, T4).

There appear to be three ways to understand E.P.'s considerable capacity for new learning. First, E.P. has partial sparing of the parahippocampal cortex within the medial temporal lobe, and this spared cortex might support his ability to acquire some amount of declarative knowledge. Second, with the advantage of repetition, some amount of declarative knowledge might be acquired directly by the neocortex, and independently of the medial temporal lobe, as discussed for example by Tulving (1991). Third, E.P.'s ability to improve his performance might depend on some form of nondeclarative memory that is either intrinsic to neocortex or supported by some other brain system.

Our results strongly indicate that E.P.'s learning depended on nondeclarative memory. First, his ability to complete the threeword sentences was not accompanied by conscious knowledge. At no time did he indicate awareness that he was sometimes producing correct answers, and during memory testing he never acknowledged that the test material was familiar. Furthermore, the confidence ratings that he assigned to his correct answers were the same as the confidence ratings that he assigned to his incorrect answers.

Second, the pattern of E.P.'s response times in the forcedchoice test differed from what is ordinarily observed when recognition memory is tested. Typically, for individuals who perform above chance, response times are faster for correct than for incorrect responses (Pike et al., 1977). However, E.P.'s response times were nearly identical for correct and incorrect responses, suggesting that his responses were outside of awareness. To our knowledge, this is the first report of identical correct and incorrect response times in an individual performing significantly above chance levels on a memory test.

Third, the information acquired by E.P. differed qualitatively from the information acquired by controls. Controls acquired knowledge that was flexible and resistant to modifications in the test stimuli. Thus, when the second word in the sentence frame was replaced by a synonym (or when both the first and second words were replaced), the performance of controls on the cued recall test was not measurably affected (28.7\% correct vs $24.7 \%$ correct after synonym replacement). In sharp contrast, E.P.'s performance fell from $20.7 \%$ correct to $3.4 \%$ correct (one item correct) after synonym replacement. Thus, E.P. exhibited extreme sensitivity to modifications of the test stimuli and appeared able to access what he had learned only when the test items appeared exactly as they had at study.

The same principle may explain why on the long-term retention test E.P. and the less extensively trained controls performed similarly on the cued-recall test but dissimilarly on the forcedchoice test. Thus, on the cued-recall test, E.P. scored $10.4 \%$ correct (three months after his 24 training sessions), and controls scored $7.6 \%$ correct (five to 6 months after their two training sessions). In contrast, on the forced-choice test E.P. scored only $57.3 \%$ correct (controls, $81.3 \%$ correct). Thus, the controls were able to express what they had learned in both the cued-recall test and in the simpler forced-choice test. However, E.P. was rela- tively better at the cued-recall test, perhaps because the format and the requirements of that test more closely resembled the format and the requirements of the training sessions themselves. This relative inflexibility of acquired information is considered an important characteristic of nondeclarative memory (Cohen, 1984; Squire, 1992; Eichenbaum and Cohen, 2001).

A number of studies have documented that memory-impaired patients can acquire new factual (semantic) knowledge, given sufficient repetition of what is to be learned (Shimamura and Squire, 1988; Hamann and Squire, 1995; Vargha-Khadem et al., 1997; Kitchener et al., 1998; Reed and Squire, 1998; Verfaellie et al., 2000; Van der Linden et al., 2001; Westmacott and Moscovitch, 2001). However, in these cases the residual learning ability in amnesia appears to result in declarative knowledge. The present findings provide evidence for the acquisition of information by nondeclarative memory, information that would be learned declaratively (as facts) by normal individuals as well as by amnesic patients, so long as they are not too severely impaired. Notably, the learning that E.P. exhibited did not result in factual knowledge in the usual sense of that term, although successful learning of this material would ordinarily be expected to result in some amount of knowledge. Instead, what E.P. learned was available only through performance, and information was expressed in the absence of any awareness that memory was being used.

What kind of nondeclarative memory might have supported E.P.'s gradual learning? The phenomenon of priming (Tulving and Schacter, 1990) is thought to result in improved perceptual fluency and to bias performance independently of conscious recollection. Yet, priming is ordinarily associated with single-trial paradigms, not prolonged learning. Moreover, there is little evidence that priming can influence forced-choice judgments (Hamann and Squire 1997; Stark and Squire, 2000). Habit learning is a more plausible alternative. Habit learning develops gradually and depends on the integrity of the neostriatum (Mishkin and Petri, 1984; Tulving, 1991; Knowlton et al., 1996; Teng et al., 2000; Fernandez-Ruiz et al., 2001). However, the kind of habit learning that depends on the neostriatum is thought to proceed as a gradual strengthening of responses through reinforcement. In the present study, no feedback was provided during learning.

It is possible that E.P. gradually learned the three-word sentences through perceptual learning. Perceptual learning is intrinsic to the neocortex and involves learning about stimuli and their attributes through extended exposure (Gilbert et al., 2001). Typically, perceptual learning is highly specific to the stimuli being learned and does not transfer to stimuli presented in a different form or orientation. In the present study what E.P. learned was represented more abstractly than one would expect in the case of typical perceptual learning, because what he learned could accommodate either spoken or visual presentation of the test material. At the same time, the learning did exhibit stimulus specificity that is characteristic of perceptual learning, inasmuch as the learning could not accommodate modifications in the stimuli themselves. We suggest that the improved performance exhibited by E.P. occurred as the result of direct learning by the neocortex, independently of the medial temporal lobe, and by a process akin to perceptual learning. It seems unlikely that remaining tissue in the posterior parahippocampal cortex is important, because E.P. has no detectable capacity for declarative memory, and there is no evidence linking this region exclusively to nondeclarative forms of memory.

The present findings for E.P. can be usefully considered in the context of what has been reported for a different patient (K.C.) in 
earlier pioneering studies of fact learning in amnesia (Tulving et al., 1991; Hayman et al., 1993). K.C. developed memory impairment after a head injury, which damaged his medial temporal lobe bilaterally. His damage is asymmetric and present in only a small portion of the right medial temporal lobe. Moreover, the damage also involves left frontal, left parietal, left retrosplenial, and left occipital cortex, and there is a small lesion in right parietal cortex (Tulving et al., 1991). K.C. was taught responses to short phrases following the same procedure as in the present study (i.e., very similar schedule of pretest and training sessions and the same number of test items presented with the study-only procedure) (Hayman et al., 1993). After 4 weeks of training and 16 training trials, K.C. scored $83 \%$ correct on a cued-recall test, whereas E.P. scored only $2.1 \%$ correct after 4 weeks of training and 16 trials (Fig. 1, T1). Thus, E.P,'s capacity for learning is much poorer than K.C.'s capacity and is apparent only after more extended training. This difference is likely related to E.P.'s more extensive medial temporal lobe damage. Accordingly, K.C. may succeed to a greater extent than E.P. because K.C. has less medial temporal lobe damage and is less amnesic than E.P. It is interesting to note that K.C., like E.P., exhibited cross-modal transfer of what he had learned. However, it was not otherwise determined what kind of knowledge K.C. acquired, that is, whether his new knowledge was similar to or different from the declarative knowledge about the world that he had acquired before his amnesia (Hayman et al., 1993).

A more recent study of postmorbid fact learning and vocabulary learning by K.C. concluded that he acquired declarative (explicit) knowledge that was consciously accessible but that the knowledge was incomplete, inefficient, and relatively inflexible, perhaps as a consequence of the information having been acquired directly by neocortex (Westmacott and Moscovitch, 2001). It may also be useful to explore an alternative possibility: that K.C.'s learning was supported by structures within the medial temporal lobe that remain intact and that the characteristics of his knowledge, rather than being unusual and qualitatively distinct, are what one might observe in any individual who has only partly learned a body of material.

It is also useful to note, considering that K.C. has less medial temporal lobe damage than E.P. (and does better at new learning), that K.C.'s medial temporal lobe damage cannot explain instances where he performs more poorly than E.P. For example, it is notable that K.C. is reported to be able to recall very few, if any, autobiographical episodes from his life before his injury (Tulving et al., 1988; Hayman et al., 1993; Moscovitch et al., 2000; Westmacott et al., 2001). In contrast, E.P. can recall autobiographical episodes from his early life in considerable detail (Reed and Squire, 1998; Bayley and Squire, 2001).

Thus, the findings from K.C. and E.P. constitute a double dissociation with respect to autobiographical recollection (better for E.P.) and the ability to acquire new information in anterograde amnesia (better for K.C.). These findings suggest that the difficulties reported for K.C. in autobiographical recollection are not related to his medial temporal lobe damage (because E.P. has extensive medial temporal lobe damage and yet can recollect autobiographical episodes better than K.C.). Thus, contrary to recent discussions (Moscovitch et al., 2000; Rosenbaum et al., 2000; Westmacott et al., 2001), K.C.'s performance on tests of retrograde amnesia for personal events is unlikely to illuminate the function of the hippocampus or other medial temporal lobe structures.

In summary, despite extensive medial temporal lobe damage,
E.P. was gradually able to acquire new information. In contrast to what might have been expected, this information was not represented as new knowledge available to conscious recollection. Rather, the information that he acquired was nondeclarative and was not experienced as factual information. It was outside of awareness, it could be expressed only through performance, and it was relatively inflexible, accessible only when precisely the same cues were used to test memory that had been present during study. The results show that factual material, which is ordinarily learned declaratively and with the participation of the medial temporal lobe, can also be acquired as nondeclarative memory. We propose that in those cases when factual information is acquired as declarative knowledge, for example, when factual knowledge is slowly acquired as conscious knowledge by memoryimpaired patients, then intact structures within the medial temporal lobe are responsible for the learning. In contrast, when such information is acquired as nondeclarative knowledge, as in the present study, we propose that the learning occurs directly within neocortex.

\section{REFERENCES}

Baddeley A, Vargha-Khadem F, Mishkin M (2001) Preserved recognition in a case of developmental amnesia; implications for the acquisition of semantic memory. J Cognit Neurosci 13:357-369.

Bayley PJ, Squire LR (2001) Detailed recall of remote autobiographical memories in amnesia. Soc Neurosci Abstr 27:909.

Cohen NJ (1984) Preserved learning capacity in amnesia: Evidence for multiple memory systems. In: Neuropsychology of memory (Squire LR, Butters N, eds), pp 83-103. New York: Guilford.

Eichenbaum H, Cohen NJ (2001) From conditioning to conscious recollection: memory systems of the brain. New York: Oxford UP.

Eichenbaum H, Mathews P, Cohen NJ (1989) Further studies of hippocampal representation during odor discrimination learning. Behav Neurosci 103:1207-1216.

Eichenbaum H, Stewart C, Morris RGM (1990) Hippocampal representation in place learning. J Neurosci 10:3531-3542.

Fernandez-Ruiz J, Wang J, Aigner T, Mishkin M (2001) Visual habit formation in monkeys with neurotoxic lesions of the ventrocaudal neostriatum. Proc Natl Acad Sci USA 98:4196-4201.

Gabrieli J, Cohen N, Corkin S (1988) The impaired learning of semantic knowledge following bilateral medial temporal-lobe resection. Brain Cognit 7:157-177.

Gilbert CD, Sigman M, Crist RE (2001) The neural basis of perceptual learning. Neuron 31:681-697.

Glisky EL, Schacter DL, Tulving E (1986a) Computer learning by memory-impaired patients: acquisition and retention of complex knowledge. Neuropsychologia 24:313-328.

Glisky EL, Schacter DL, Tulving E (1986b) Learning and retention of computer-related vocabulary in memory-impaired patients: method of vanishing cues. J Clin Exp Neuropsychol 8:292-312.

Hamann SB, Squire LR (1995) On the acquisition of new declarative knowledge in amnesia. Behav Neurosci 109:1027-1044.

Hamann SB, Squire LR (1997) Intact perceptual memory in the absence of conscious memory. Behav Neurosci 111:850-854.

Hayman CA, MacDonald CA, Tulving E (1993) The role of repetition and associative interference in new semantic learning in amnesia: a case experiment. J Cognit Neurosci 5:375-389.

Hood KL, Postle BR, Corkin S (1999) An evaluation of the concurrent discrimination task as a measure of habit learning: performance of amnesic subjects. Neuropsychologia 37:1375-1386.

Kitchener EG, Hodges JR, McCarthy R (1998) Acquisition of postmorbid vocabulary and semantic facts in the absence of episodic memory. Brain 121:1313-1327.

Knowlton BJ, Mangels JA, Squire LR (1996) A neostriatal habit learning system in humans. Science 273:1399-1402.

Kritchevsky M, Squire LR, Zouzounis JA (1988) Transient global amnesia: characterization of anterograde and retrograde amnesia. Neurology 38:213-219.

Marslen-Wilson WD, Teuber HL (1975) Memory for remote events in anterograde amnesia: recognition of public figures from news photographs. Neuropsychologia 13:353-364.

Milner B, Corkin S, Teuber HL (1968) Further analysis of the hippocampal amnesic syndrome: 14-year follow-up study of $\mathrm{H}$. M. Neuropsychologia 6:215-234.

Milner B, Squire LR, Kandel ER (1998) Cognitive neuroscience and the study of memory. Neuron 20:445-468.

Mishkin M, Petri HL (1984) Memories and habits: Some implications 
for the analysis of learning and retention. In: Neuropsychology of memory (Squire LR, Butters N, eds), pp 287-296. New York: Guilford. Moscovitch M, Yaschyshyn T, Ziegler M, Nadel L (2000) Remote episodic memory and retrograde amnesia: was Endel Tulving right all along? In: Memory, consciousness and the brain: the Tallinn Conference (Tulving E, ed), pp 331-345. Philadelphia, PA: Psychology Press/ Taylor and Francis.

Osterrieth PA (1944) Le test de copie d'une figure complexe. Archives de Psychologie 30:206-356.

Pike R, Dalgleish L, Wright J (1977) A multiple-observations model for response latency and the latencies of correct and incorrect responses in recognition memory. Mem Cognit 5:580-589.

Reed JM, Squire LR (1998) Retrograde amnesia for facts and events: findings from four new cases. J Neurosci 18:3943-3954.

Reed JM, Hamann SB, Stefanacci L, Squire LR (1997) When amnesic patients perform well on recognition memory tests. Behav Neurosci 111:1163-1170.

Rey A (1964) L'examen clinique en psychologie. Paris: Presses Universitaires de France.

Rosenbaum DL, Priselac S, Kohler S, Black S, Gao F, Nadel L, Moscovitch M (2000) Remote spatial memory in an amnesic person with extensive bilateral hippocampal lesions. Nat Neurosci 3:1044-1048.

Saunders RC, Weiskrantz L (1989) The effects of fornix transection and combined fornix transection, mammillary body lesions and hippocampal ablations on object-pair association memory in the rhesus monkey. Behav Brain Res 35:85-94.

Scoville WB, Milner B (1957) Loss of recent memory after bilateral hippocampal lesions. J Neurol Neurosurg Psychiat 20:11-21.

Shimamura AP, Squire LR (1988) Long-term memory in amnesia: cued recall, recognition memory, and confidence ratings. J Exp Psychol Learn Mem Cogn 14:763-770.

Squire LR (1992) Memory and the hippocampus: a synthesis from findings with rats, monkeys, and humans. Psychol Rev 99:195-231.

Squire LR, Shimamura AP (1986) Characterizing amnesic patients for neurobehavioral study. Behav Neurosci 100:866-877.

Stark CEL, Squire LR (2000) Recognition memory and familiarity judgments in severe amnesia: no evidence for a contribution of repetition priming. Behav Neurosci 114:459-467.

Stefanacci L, Buffalo EA, Schmolck H, Squire LR (2000) Profound amnesia following damage to the medial temporal lobe: a neuroanatomical and neuropsychological profile of patient E.P. J Neurosci 20:7024-7036.

Teng E, Stefanacci L, Squire LR, Zola SM (2000) Contrasting effects on discrimination learning following hippocampal lesions or conjoint hippocampal-caudate lesions in monkeys. J Neurosci 20:3853-3863.

Thorndike EL, Lorge I (1944) The Teacher's Wordbook of 30,000 Words. New York: Columbia University Teachers College, Bureau of Publications.

Tulving E (1991) Concepts of human memory. In: Memory: organization and locus of change (Squire L, Weinberger N, Lynch G, McGaugh J, eds), pp 3-32. New York: Oxford UP.

Tulving E, Schacter DL (1990) Priming and human memory systems. Science 247:301-306.

Tulving E, Schacter DL, McLachland D, Moscovitch M (1988) Priming of semantic autobiographical knowledge: a case study of retrograde amnesia. Brain Cognit 8:3-20.

Tulving E, Hayman CAG, MacDonald CA (1991) Long-lasting perceptual priming and semantic learning in amnesia: a case experiment. J Exp Psychol Learn Mem Cogn 17:595-617.

Van der Linden M, Cornil V, Meulemans T, Ivanoiu A, Salmon E, Coyette F (2001) Acquisition of a novel vocabulary in an amnesic patient. Neurocase 7:283-293.

Vargha-Khadem F, Gaffan D, Watkins KE, Connelly A, Van Paesschen W, Mishkin M (1997) Differential effects of early hippocampal pathology on episodic and semantic memory. Science 277:376-380.

Verfaellie M, Reiss L, Roth HL (1995) Knowledge of new English vocabulary in amnesia: an examination of premorbidly acquired semantic memory. J Int Neuropsychol Soc 1:443-453.

Verfaellie M, Koseff P, Alexander MP (2000) Acquisition of novel semantic information in amnesia: effects of lesion location. Neuropsychologia 38:484-492.

Warrington EK (1984) Recognition memory test. Windsor, UK: NFER-Nelson.

Wechsler D (1987) Wechsler memory scale-revised manual. San Antonio, TX: The Psychological Corporation.

Wechsler D (1997) Wechsler adult intelligence scale-third edition. San Antonio, TX: The Psychological Corporation.

Westmacott R, Moscovitch M (2001) Names and words without meaning: incidental postmorbid semantic learning in a person with extensive bilateral medial temporal lobe damage. Neuropsychology 15:586-596.

Westmacott R, Leach L, Freedman M, Moscovitch M (2001) Different patterns of autobiographical memory loss in semantic dementia and medial temporal lobe amnesia: a challenge to consolidation theory. NeuroImage 7:37-55.

Wilson B (1992) Rehabilitation and memory disorders. In: Neuropsychology of memory, Ed 2 (Squire L, Butters N, eds), pp 315-321. New York: Guilford. 